\title{
SPORTWISSENSCHAFTLICHER HOCHSCHULTAG 82
}

Die Deutsche Vereinigung für Sportwissenschaft (DVS) veranstaltet vom 6. Oktober 1982, $15.00 \mathrm{Uhr}$, bis 8. Oktober 1982, 13.00 Uhr, in Zusammenarbeit mit dem Institut für Sportwissenschaft der Universität Tübingen den SPORTWISSENSCHAFTLICHEN HOCHSCHULTAG 1982 unter dem Thema

Hauptvorträge

$$
\text { SPIEL - SPIELE - SPIELEN }
$$

H. Bausinger: Spiel unter Dummen

O. Grupe: Das Spiel - eine Rahmenanalyse

L. Krappmann: Sozialisation im Spiel

B. Sutton-Smith: The Idealisation of play

Arbeitskreise und Sektionen mit einführenden Referaten und Kurzreferaten

Spieltheorien im Wandel; Ref.: H. Scheuerl, Ltg.: A. Flitner.

Diskussion der Referate Grupe und Sutton-Smith; Ltg.: G. Anders.

Freizeit und Spiel; Ref.: G. Eichler, Ltg.: A. Trebels.

Spiel in handlungstheoretischer Sicht. Methodische Ansätze in der Spielforschung;

Ref.: G. Kaminski, Ltg.: K. Willimczik.

Sportspielspezifische Vermittlungsmodelle; Ref.: K. Dietrich, Ltg.: F. Begov.

Kulturgeschichte des Spiels; Ref.: H. Eichberg. Diskussion des Ref. Bausinger;

Ltg.: H. Denk.

Psychoanalytische Aspekte des Spiels; Ref.: G. Bittner, Itg.: R. Fatke.

Psychologische Aspekte des Sportspiels; Ref.: H. Eberspächer, Ltg.: K. Kohl.

Sportspielbeobachtung; Ref.: G. Hagedorn, Ltg.: R. Andresen.

Sportspielspezifische Vermittlungsmodelle am Beispiel Tennis;

Ltg.: M. Kleine-Tebbe.

Wie regeln Spielregeln das Spiel? Ref.: G. Gebauer, Ltg.: H. Digel.

Diskussion des Referats Krappmann; Ltg. C. Kleindienst-Cachay.

Spiel und Umwelt; Ref.: K. H. Scherler, Ltg.: W. Preising.

New Games und die Spielbewegung des DSB;

Ref.: P. Kapustin / W. Platzek, Ltg.: H. Röhrs.

Integrative Spielvermittlungsmodelle in der Sportlehrerausbildung;

Ref.: W. D. Brettschneider, Ltg.: H. J. Schaller.

$\mathrm{Zu}$ den Arbeitskreisen und Sektionen können Kurzreferate (10 Min.) angemeldet werden. Kongreßteilnehmer, die dies möchten, reichen eine Kurzfassung ihres Vortrags (ca. 1-2 Seiten) bis spätestens 1. Juli beim Organisationsausschuß ein. Uber die Annahme des Referats entscheiden DVS-Vorstand und Organisationsausschuß bis Mitte Juli.

Im Rahmen des Hochschultages sind kleinere Ausstellungen und Vorführungen vorgesehen; außerdem wird den Teilnehmern Gelegenheit geboten, sich selbst spielerisch zu betätigen: New Games mit P. Kapustin/W. Platzek, Tischtennis mit M. Grumbach, Tanzspiele mit I. Mißmahl, Volleyball mit F. Begov, Tennis mit H. Gabler.

Die Kongreßgebühr beträgt für DVS-Mitglieder 70,- DM, für Nichtmitglieder 120,DM und für Studierende 35,- DM. Überweisungen auf Konto Nr. 169099 bei der Kreissparkasse Tübingen, BLZ 64150020 , Kennwort „Sportwissenschaftlicher Hochschultag" bis zum 6. 9. 1982; danach erhöht sich die Gebühr um 20,- DM.

Anmeldungen sind $z u$ richten an den Organisationsauschuß „Sportwissenschaftlicher Hochschultag 1982“, Institut für Sportwissenschaft, Wilhelmstraße 124, 7400 Tübingen.

$\mathrm{Da}$ Tübingen über keine sehr große Hotelkapazität verfügt, wird dringend empfohlen, sich sofort mit dem Verkehrsverein, Eberhardsbrüdke 1 (Stichwort Sportwissenschaftlicher Hochschultag), betr. Zimmerreservierung in Verbindung zu setzen.

DVS-Vorstand

Ortl. Organisationsausschuß 